\title{
Analyse monosomique de la résistance à Septo- ria nodorum Berk chez le blé tendre (Triticum aestivum L. em. Thell (Vill) M.K.)
}

\author{
Philippe AURIAU, Frantz RAPILLY $\left({ }^{*}\right)$ \& Yvonne CAUDERON \\ avec la collaboration technique de Hervé Richard) (*), Joséphine Roussel \& Camille Depaturi:aux \\ I.N.R.A., Station de Génétique et d'Amélioration des Plantes. \\ (*) Station de Pathologie Végétale, Centre de Recherches de Versailles, route de Saint-Cyr, F 78000 Versailles
}

Les 21 lignées monosomiques du c $v$ « Courtot » ont été croisées par la lignée « L 22 », résistante a la septoriose, utilisée comme pollinisatrice. Les plantes $\mathrm{F} 1$ monosomiques et disomiques obtenues ont été repiquees avec la lignée " L 22 » dans un essai randomisé, qui a été contaminé artificiellement à la fin mai. Les principaux résultats obtenus figurent au tableau 1.

La durée d'incubation, ou LPI, a permis de distinguer de la F1 disomique, les $\mathrm{F} 1$ monosomiques $3 \mathrm{~A}, 4 \mathrm{~A}, 2 \mathrm{~B}$, $5 \mathrm{~B}, 2 \mathrm{D}, 7 \mathrm{D}$ plus résistantes et les $\mathrm{F} 1$ monosomiques $1 \mathrm{~A}, 3 \mathrm{~B}, 6 \mathrm{~B}, 5 \mathrm{D}, 6 \mathrm{D}$ plus sensibles.

L'intensité de maladie, déterminée en début juillet, ou $\mathrm{P}$ max, a permis de distinguer de la $\mathrm{F} 1$ disomique, les $\mathrm{Fl}$ mosomiques $3 \mathrm{~A}, 4 \mathrm{~A}, 5 \mathrm{~A}, 6 \mathrm{~A}, \mathrm{~B}, 2 \mathrm{~B}, 5 \mathrm{~B}, 7 \mathrm{~B}, 2 \mathrm{D}$ et la lignée « $\mathrm{L} 22$ » plus résistantes, et les $\mathrm{F} 1$ monosomiques 6B, 3D, 4D, 6D plus sensibles.

D'autre part si l'on considère la figure 2, la droite de régression générale de $\mathrm{P}$ max sur LPI a pour équation :

$$
\mathrm{P} \max =14,56+0,72 \mathrm{LPI}, \mathrm{r}=0,57 \mathrm{HS}
$$

un tiers seulement des variations de $\mathrm{P}$ max sont expliquées par celles de LPI.

Mais on peut aussi regrouper les entrées en trois groupes, correspondant à peu près aux diagonales du tableau 2:

groupe 1: F1 monosomiques 3A, 5A, 6A, 1B, 3B, 7B et la lignée « L 22 »

groupe 2: F1 monosomiques 1A, 2A, 4A, 7A, 2B, 4B, 5B, 6B, 1D, 2D, 3D, 5D, 6D et la F1 disomique groupe $3: \mathrm{Fl}$ monosomiques $4 \mathrm{D}$ et $7 \mathrm{D}$.

Les pentes des droites de régression des deux premiers groupes ne sont pas significativement différentes entre elles, ni avec la pente de la droite générale de régression.

Par contre les ordonnées à l'origine des droites des groupes 1 et 2 sont différentes, la valeur de cette différence hautement significative est très proche de la différence entre les valeurs de $\mathrm{P}$ max pour la lignée « $\mathrm{L} 22$ » et la F1 disomique.

A ces trois groupes correspondraient les chromosomes de la lignée « L 22 » porteurs de gènes de résistance ou de sensibilité au niveau de P max, à LPI constante.

La plus grande résistance de la Fl monosomique 3A pour les deux paramètres pourrait être utile en sélection. Enfin des différences ont aussi été observées pour la précocité de floraison, la longueur des tiges et les poids de 1000 grains. Mais aucune relation n'a été trouvée entre ces différences et le niveau de résistance.

Mots clés additionnels : Analyse monosomique, résistance partielle, Septoriose, Septoria nodorum, Triticum aestivum.

The common wheat line "L 22", fairly resistant to glume blotch, was used as the pollinator in crosses on the 21 different monosomic lines of "Courtot". All the monosomic F1 plants obtained, as well as the disomic ones, were grown with plants of line " $L 22$ " in a completely randomized trial, then artificially infected in late May. The results obtained are presented in table 1 .

Compared to the disomic F1 the monosomic for 3A, 4A, 2B, 5B, 2D, 7D and 1A, 3B, 6B, 5D, 6D had an incubation time (LPI) increased (more resistance) or reduced (more susceptibility), respectively. Similarly the monosomic F1, 3A, 4A, 5A, 6A, 1B, 2B, 5B, 7B, 2D and line " $\mathrm{L} 22$ " showed a disease intensity ( $\mathrm{P}$ max) reduced (more resistance) in early July, while monosomic F1 6B, 3D, 4D, 6D showed more susceptibility at this stage. The general regression line of $P$ max on LPI (fig. 2) was :

$$
P \max =14.56+0.72 \mathrm{LPI}, \mathrm{r}=0.57 \mathrm{HS}
$$

thus a third of the variation of $\mathrm{P}$ max was related to that of LPI.

The three following groups could be constituted:

group 1:3A, 5A, 6A, 1B, 3B, 7B and line "L 22"

group $2: 1 \mathrm{~A}, 2 \mathrm{~A}, 4 \mathrm{~A}, 7 \mathrm{~A}, 2 \mathrm{~B}, 4 \mathrm{~B}, 5 \mathrm{~B}, 6 \mathrm{~B}, 1 \mathrm{D}, 2 \mathrm{D}, 3 \mathrm{D}, 5 \mathrm{D}, 6 \mathrm{D}$ and the disomic $\mathrm{F} 1$

group $3: 4 \mathrm{D}, 7 \mathrm{D}$. 
The slopes of the regression lines for groups 1 and 2 were neither significantly different from each other, nor from that of the general regression. But the difference between their intercepts was highly significant, and comparable to the difference of $\mathrm{P}$ max values between line " $\mathrm{L} 22$ " and the disomic F1. Differences in $\mathrm{P}$ max values, at fixed LPI, could correspond to three groups of chromosomes line "L 22", carrying genes for resistance or susceptibility. The high resistance of the $\mathrm{Fl}$ monosomic $3 \mathrm{~A}$ for both parameters could be useful for breeding purposes. Differences were also observed for stem lengths, flowering date and thousand kernel weight but no relation was observed between these differences and the level of resistance observed.

Additional key words : Monosomic analyses, partial resistance, glume blotch, Septoria nodorum, Triticum aestivum.

\section{INTRODUCTION}

Des travaux antérieurs (RAPILLY et al., 1981, 1984) ont montré qu'il est possible de sélectionner des lignées de blé tendre présentant des niveaux différents de résistances partielles à la septoriose. Ces niveaux peuvent être caractérisés par l'observation in situ de l'expression de 2 séquences épidémiques, la durée d'incubation LPI qui s'achève lorsqu'apparaissent les premiers symptômes chlorotiques, et la vitesse d'extension des nécroses foliaires $P$ max.

L'interprétation des disjonctions observées suggérait que LPI et $\mathrm{P}$ max avaient un déterminisme génétique complexe.

Une relation avait été trouvée entre la longueur des tiges ou la précocité d'épiaison et le degré d'expression de ces résistances partielles. Ce résultat concordait avec certaines observations de TROTTET \& MERRIEN (1982). Pour tenter d'éclaircir ces différents points une analyse monosomique de la LPI et de P max a été entreprise sur F1 en 1985-1986.

\section{MATÉRIEL ET MÉTHODES}

\section{A. Matériel végétal}

L'analyse a porté sur la lignée résistante « $\mathrm{L} 22$ » caractérisée par une longue durée d'incubation (LPI) et une faible vitesse d'extension des symptômes ( $\mathrm{P}$ max). Cette lignée est issue du croisement « Carala »/« Roazon »//« Carala», où la source de résistance est «Carala » (RAPILly et al., 1984).

La série monosomique utilisée est celle de "Courtot » obtenue par une suite de rétrocroisements réalisés à Versailles et Clermont-Ferrand (DENIS et al., 1982) sur la série monosomique de «Chinese spring » isolée par SEARS (1954).

Des croisements ont été réalisés entre les 21 lignées monosomiques de "Courtot " et la lignée «L 22 » utilisée comme pollinisatrice.

Pour chacun de ces 21 croisements 12 grains germés en boîtes de Pétri ont fait l'objet d'un dénombrement chromosomique sur pointes de racines.

Les racines prélevées ont été prétraitées à l' $\alpha$-monobromonaphtalène pendant $15 \mathrm{~h}$ à une température de $5{ }^{\circ} \mathrm{C}$, puis fixées à l'acide acétique à $90 \mathrm{p} .100$ pendant une demi-heure, et stockées dans l'éthanol à $70^{\circ}$ pendant au moins une semaine.
Elles ont ensuite été colorées au réactif de Schiff pendant $1 \mathrm{~h}$ après une hydrolyse de $12 \mathrm{mn}$ à $60^{\circ} \mathrm{C}$.

Après écrasement, entre lame et lamelle, des pointes de racines dans une goutte de carmin acétique, l'observation au microscope a permis de distinguer les plantes monosomiques à 41 chromosomes, des plantes disomiques à 42 chromosomes.

\section{B. Dispositif expérimental}

Après dénombrement chromosomique les jeunes plantes ont été mises en pots et ont passé l'hiver à l'abri des gelées dans un châssis. Au mois de mars elles ont été repiquées dans un essai constitué de 12 lignes jumelées. L'intervalle entre les lignes était de $20 \mathrm{~cm}$ ou de $40 \mathrm{~cm}$ et sur la ligne entre les plantes de $15 \mathrm{~cm}$, ceci pour faciliter les observations plante à plante.

La répartition sur le terrain des plantes mono ou disomiques entre lignes était faite au hasard : chaque ligne de 24 plantes comportait aussi une plante de la lignée " $\mathrm{L} 22$ » comme témoin de résistance et une plant « de chacune des 2 variétés « Roazon » et «Courtot » (les plantes de Courtot ont dû être arrachées à cause de leur sensibilité à la rouille jaune), comme témoins de la réussite de la contamination.

\section{Inoculation}

La souche 6 de Septoria nodorum isolée et multipliée sur la variété de blé tendre "Etoile de Choisy » a été utilisée.

La contamination a été réalisée les 21 et 30 mai 1986 par pulvérisation sur le feuillage d'une suspension de spores contenant environ $10^{6}$ spores par millilitre.

\section{Notation et interprétation des résultats}

La durée d'incubation (LPI) a été évaluée plante à plante par 5 notations échelonnées entre le 20 juin et le 4 juillet. L'incubation est considérée comme achevée lorsqu'on observe l'apparition des premières chloroses foliaires. Des passages ultérieurs permettent de contrôler que ces chloroses évoluent en symptômes typiques de septoriose.

Une représentation graphique de l'évolution du pourcentage de plantes malades en fonction du temps a permis de comparer les FI monosomiques à la moyenne des disomiques (fig. 1). Les aires comprises 

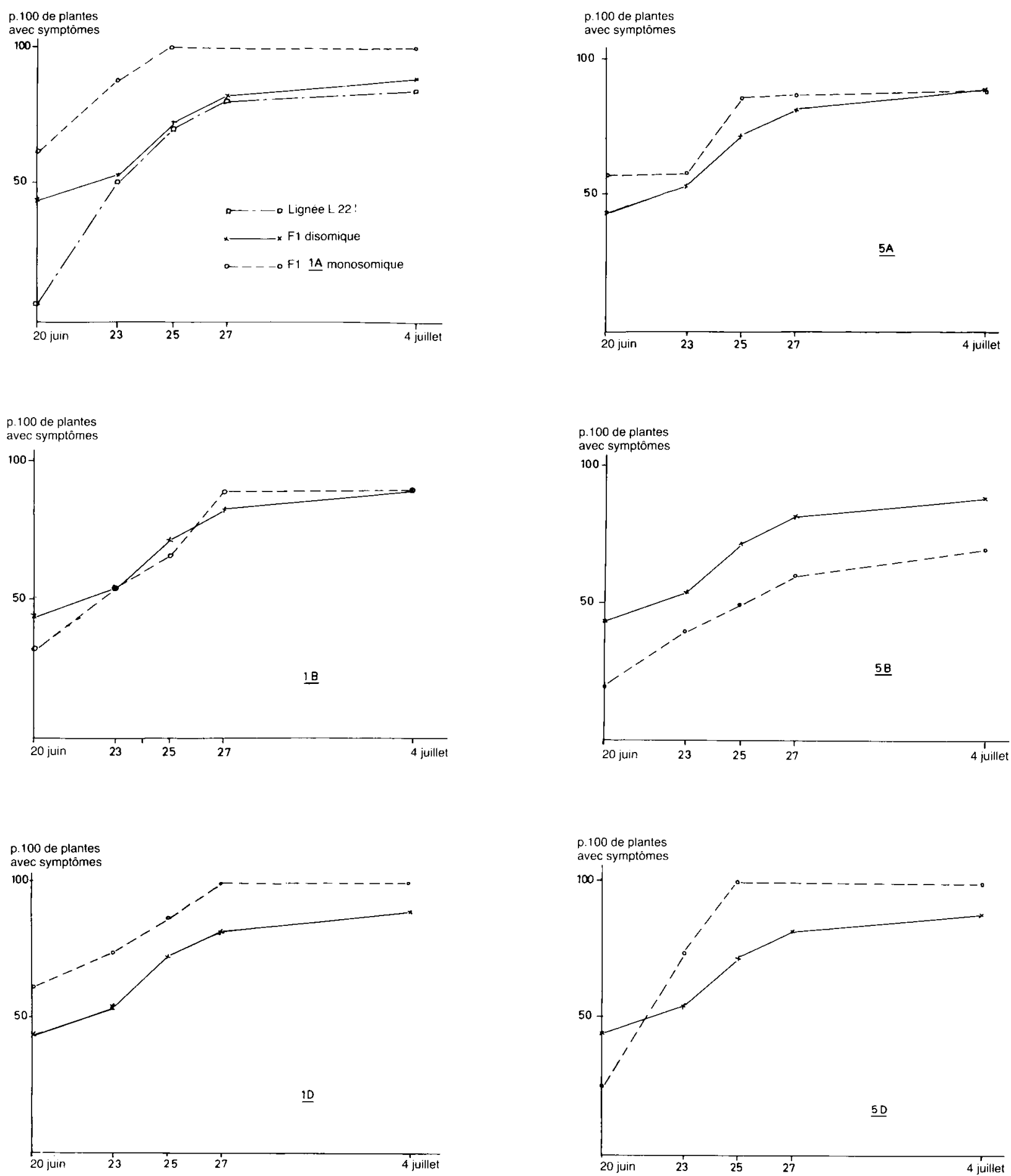

Figure 1

Exemples des courbes des durées d'incubation en fonction du temps pour 2 groupes d'homéologie.

Examples of the curves of the variation of LPI with time for two groups of homeology.

entre ces courbes et l'axe des abcisses constituent la meilleure appréciation de la LPI ; ce sont elles qui figurent au tableau 1.

Une notation d'intensité de la maladie a été faite le 7 juillet et a permis de déterminer les plantes pour lesquelles la surface des derniers étages foliaires était nécrosée à $50 \mathrm{p} .100$ au moins.

Comme les symptômes observés étaient tous issus des contaminations artificielles, la densité des lésions a peu varié. En raison de la durée séparant l'apparition

des symptômes de la date de cette notation, l'intensité de la maladie a surtout dépendu de la vitesse d'extension des lésions ( $P$ max) qui a ainsi été globalement évaluée.

Enfin on a aussi, et pour chaque plante, déterminé la date de floraison, la hauteur des tiges et le poids de 1000 grains sur l'ensemble des épis laissés en fécondation libre. En effet les 2 épis ensachés sur chaque plante ont fourni des grains échaudés du fait même de ce traitement. 
TABLEAU 1

Résultats obtenus sur la lignée « $L 22$ », les 21 Fl monosomiques et la Fl disomique du croisement « Courtot »/《L $22 »$. (I) En pourcentage des effectifs.

(2) En pourcentage de la Fl disomique.

Les symboles $1 A, 5 B, 2 D$ etc... correspondent aux chromosomes pour lesquels les FI étudiées sont monosomiques.

Results obtained on the monosomic and disomic F1 of the cross " Courtot "/ L $L 22$ » and on the line « L 22 " itself. (1) Percentage of the total number for each monosomic Fl.

(2) Percentage relative to the disomic Fl.

The symbols $1 A, 5 B, 2 D$ etc... correspond to the chromosomes for which the different $F 1$ are monosomic.

\begin{tabular}{|c|c|c|c|c|c|c|c|}
\hline \multirow[t]{2}{*}{ F1 } & \multirow[t]{2}{*}{$\begin{array}{c}\text { Nombre } \\
\text { de plantes }\end{array}$} & \multirow[t]{2}{*}{$\begin{array}{l}\text { LPI appréciée par l'aire } \\
\text { sous la courbe en } \% \\
\text { de celle des disomiques }\end{array}$} & \multicolumn{2}{|c|}{$\begin{array}{l}\text { Plantes ayant au moins la } \\
\text { moitié du feuillage nécrosé } \\
\text { (P max) (notation du 7.7.86) }\end{array}$} & \multirow[t]{2}{*}{$\begin{array}{l}\text { Hauteur } \\
\text { des pailles } \\
\text { en cm }\end{array}$} & \multirow[t]{2}{*}{$\begin{array}{l}\text { Précocité en } \\
\text { jours depuis } \\
\text { le } 1^{\text {er juin }}\end{array}$} & \multirow[t]{2}{*}{$\begin{array}{l}\text { Poids de } 1000 \\
\text { grains en g } \\
\text { (fécondation libre) }\end{array}$} \\
\hline & & & (1) & (2) & & & \\
\hline $1 \mathrm{~A}$ & 8 & 128,0 & 87 & 116 & 61,0 & 11,0 & 36,0 \\
\hline $2 \mathrm{~A}$ & 5 & 89,4 & 80 & 107 & 55,6 & 14,4 & 38,9 \\
\hline $3 \mathrm{~A}$ & 8 & 69,8 & 12 & 16 & 60,5 & 14,1 & 49,0 \\
\hline $4 \mathrm{~A}$ & 6 & 76,1 & 53 & 71 & 71,3 & 12,0 & 42,6 \\
\hline $5 / \mathrm{x}$ & 7 & 107,8 & 43 & 57 & 64,4 & 14,6 & 39,7 \\
\hline $6 \mathrm{~A}$ & 10 & 108,6 & 50 & 66 & 56,5 & 15,3 & 41,2 \\
\hline $7 \mathrm{~A}$ & 6 & 98,7 & 83 & 110 & 59,2 & 13,0 & 42,4 \\
\hline $1 \mathrm{~B}$ & 9 & 104,1 & 55 & 73 & 62,6 & 10,9 & 45,6 \\
\hline $2 \mathrm{~B}$ & 7 & 81,9 & 59 & 78 & 56,3 & 14,6 & 51,2 \\
\hline $3 \mathrm{~B}$ & 10 & 161,5 & 80 & 107 & 68,4 & 13,0 & 47,6 \\
\hline $4 B$ & 5 & 112,5 & 80 & 107 & 62,0 & 12,0 & 44,2 \\
\hline $5 \mathrm{~B}$ & 10 & 71,2 & 40 & 53 & 62,0 & 13,0 & 39,5 \\
\hline $6 \mathrm{~B}$ & 6 & 133,3 & 100 & 133 & 58,8 & 13,0 & 46,8 \\
\hline $7 \mathrm{~B}$ & 7 & 110,3 & 57 & 76 & 65,7 & 16,4 & 43,8 \\
\hline $1 \mathrm{D}$ & 8 & 112,9 & 75 & 100 & 59,1 & 11,5 & 43,9 \\
\hline $2 \mathrm{D}$ & 7 & 73,8 & 53 & 71 & 57,0 & 14,4 & 47,1 \\
\hline $3 \mathrm{D}$ & 9 & 106,6 & 88 & 117 & 56,9 & 15,7 & 38,5 \\
\hline $4 \mathrm{D}$ & 8 & 91,8 & 100 & 133 & 64,5 & 12,8 & 43,9 \\
\hline $5 \mathrm{D}$ & 8 & 120,4 & 76 & 101 & 64,6 & 12,0 & 57,9 \\
\hline $6 \mathrm{D}$ & 8 & 131,6 & 100 & 133 & 63,0 & 14,8 & 40,9 \\
\hline $7 \mathrm{D}$ & 3 & 56,1 & 66 & 88 & 64,0 & 15,0 & 41,1 \\
\hline Disomiques & 95 & 100,0 & 75 & 100 & 63,9 & 12,4 & 42,0 \\
\hline Lignée «L22» & 12 & 90,4 & 34 & 45 & 61,3 & 15,0 & 36,9 \\
\hline
\end{tabular}

\section{RÉSULTATS}

Le tableau 1 résume les principaux résultats obtenus.

\section{A. Homogénéité de l'essai}

Les 24 plantes de chaque ligne ont été divisées en 4 séries successives de 6 plantes afin de tester l'homogénéité de l'essai entre lignes et entre colonnes.

Le caractère utilisé pour ce test a été le pourcentage de plantes pour lesquelles la période d'incubation était achevée le 25 juin, date la plus discriminante.

Les valeurs de $\chi^{2}$ obtenues, 7,2 pour l'effet " lignes " et 3,8 pour l'effet " colonne ", ne sont pas significatives au seuil $P=0,05$. On peut donc conclure que les différences observées ne sont pas dues à des effets pédoclimatiques.

\section{B. Durée d'incubation (LPI)}

La figure 1 donne quelques exemples des graphiques obtenus pour chacune des $21 \mathrm{~F} 1$ monosomiques comparés aux graphiques obtenus sur les moyennes de la F1 disomique et de la lignée " $\mathrm{L} 22$ ».
En comparant au seuil de 10 p. 100 les différentes F1 monosomiques à la $\mathrm{F} 1$ disomique (tabl. 2) on constate que pour 6 chromosomes $(3 \mathrm{~A}, 4 \mathrm{~A}, 2 \mathrm{~B}, 5 \mathrm{~B}, 2 \mathrm{D}, 7 \mathrm{D})$ la surface sous la courbe est réduite, ce qui correspond à une durée d'incubation augmentée et donc à une résistance accrue. Au contraire pour 5 chromosomes (1A, $3 \mathrm{~B}, 6 \mathrm{~B}, 5 \mathrm{D}, 6 \mathrm{D})$ cette surface est accrue, ce qui correspond à une résistance diminuée.

Pour ce paramètre il n'y a pas de différence significative entre la moyenne des plantes $\mathrm{F} 1$ disomiques et la moyenne des plantes de la lignée « L 22 ».

\section{Intensité de maladie (P max)}

La comparaison au seuil 10 p. 100 des F1 monosomiques à la $\mathrm{F} 1$ disomique (tabl. 2) montre que l'intensité de maladie est diminuée pour 9 chromosomes (3A, $4 \mathrm{~A}, 5 \mathrm{~A}, 6 \mathrm{~A}, 1 \mathrm{~B}, 2 \mathrm{~B}, 5 \mathrm{~B}, 7 \mathrm{~B}, 2 \mathrm{D})$, ce qui correspond à une résistance accrue. Cette intensité est au contraire augmentée pour 4 chromosomes $(6 \mathrm{~B}, 3 \mathrm{D}, 4 \mathrm{D}, 6 \mathrm{D})$, ce qui correspond à une sensibilité accrue.

Pour ce paramètre la moyenne des plantes disomiques est moins résistante que la moyenne des plantes de la lignée « L 22 ».

Seule la F1 monosomique pour le chromosome $3 \mathrm{~A}$ 


\section{TABLEAU 2}

Chromosomes impliqués dans la résistance à la septoriose (à lire de haut en bas et de gauche à droite) - en caractère gras : poids de I 000 grains supérieurs à ceux des disomiques $(P=0,1)$,

- en italiques : poids de 1000 grains inférieurs à ceux des disomiques $(P=0,1)$.

Chromosomes implicated in resistance to glume blotch - in bold, kernel weight superior to that of the disomic F1, - in italics, kernel weight inferior to that of the disomic FI.

\begin{tabular}{|c|c|c|c|c|c|c|}
\hline & & \multicolumn{5}{|c|}{ Aire sous la courbe (LPI) } \\
\hline & & $\begin{array}{l}\text { Din } \\
\text { (rési }\end{array}$ & $\begin{array}{l}\text { uée } \\
\text { ince) }\end{array}$ & & & $\begin{array}{l}\text { Augmentée } \\
\text { (sensibilité) }\end{array}$ \\
\hline \multirow{3}{*}{ 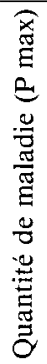 } & $\begin{array}{l}\text { Diminuée } \\
\text { (résistance) }\end{array}$ & $\begin{array}{l}3 \mathbf{A} \\
2 \mathrm{~B} \\
\mathbf{2 D}\end{array}$ & $\begin{array}{l}4 \mathrm{~A} \\
5 \mathrm{~B}\end{array}$ & & $\begin{array}{l}6 \mathrm{~A} \\
7 \mathrm{~B}\end{array}$ & \\
\hline & Egale & 7D & & $\begin{array}{l}2 \mathrm{~A} \\
\mathbf{4 B} \\
1 \mathrm{D}\end{array}$ & $7 \mathrm{~A}$ & $\begin{array}{l}1 \mathrm{~A} \\
\mathbf{3 B} \\
5 D\end{array}$ \\
\hline & $\begin{array}{l}\text { Augmentée } \\
\text { (sensibilité) }\end{array}$ & & & $3 D$ & $4 \mathrm{D}$ & $\begin{array}{l}6 \mathrm{~B} \\
6 \mathrm{D}\end{array}$ \\
\hline
\end{tabular}

est plus résistante que la lignée « L 22 » pour ce paramètre (différence significative au seuil $\mathrm{P}=0,05$ ).

\section{Caractères agronomiques (tabl. 3)}

Par rapport aux plantes $\mathrm{F} 1$ disomiques la hauteur de la tige des plantes F1 monosomiques est inférieure au seuil de 10 p. 100 pour neuf chromosomes (2A, 3A, 6A, 7A, 2B, 1D, 2D, 3D, 6D). Elle n'est augmentée que pour le chromosome $4 \mathrm{~A}$.

\section{TABLEAU 3}

Chromosomes impliqués dans la précocité de floraison et dans la hauteur des tiges (à lire de gauche à droite et de haut en bas) - en caractère gras : poids de 1000 grains supérieurs à ceux des disomiques $(P=0,1)$,

- en italiques: poids de 1000 grains inférieurs à ceux des disomiques $(P=0,1)$

Chromosomes involved in flowering earliness, stem length and thousand kernel weight

- in bold, kernel weight superior to that of the disomic FI,

- in italics, kernel weight inferior to that of the disomic Fl.

\begin{tabular}{|c|c|c|c|c|}
\hline & & \multicolumn{3}{|c|}{ Précocité de floraison } \\
\hline & & Augmentée & Egale & Diminuée \\
\hline \multirow{3}{*}{ 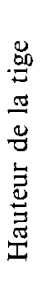 } & Augmentée & & $4 \mathrm{~A}$ & \\
\hline & Egale & $\begin{array}{l}I A \\
1 \mathrm{~B}\end{array}$ & $\begin{array}{c}3 \mathrm{~B} \text { 4B } 5 \mathrm{~B} \quad 6 \mathrm{~B} \\
4 \mathrm{D} 5 D \quad 7 \mathrm{D}\end{array}$ & $\begin{array}{l}5 \mathrm{~A} \\
7 \mathrm{~B}\end{array}$ \\
\hline & Diminuée & & $\begin{array}{l}2 \mathrm{~A} \mathbf{3 A} 7 \mathrm{~A} \\
2 \mathbf{B} \\
1 \mathrm{D}\end{array}$ & $\begin{array}{c}6 \mathrm{~A} \\
2 \mathrm{D} 3 D 6 \mathrm{D}\end{array}$ \\
\hline
\end{tabular}

Enfin la différence entre la $\mathrm{F} 1$ disomique et la lignée « L 22 »n'est pas significative.

La précocité de floraison des F1 monosomiques, comparées à la F1 disomique, est supérieure au seuil de 10 p. 100 pour les chromosomes $1 \mathrm{~A}$ et $1 \mathrm{~B}$. Elle est inférieure pour les chromosomes $5 \mathrm{~A}, 6 \mathrm{~A}, 7 \mathrm{~B}, 2 \mathrm{D}, 3 \mathrm{D}, 6 \mathrm{D}$. De même la $\mathrm{F} 1$ disomique est plus précoce que la lignée « $\mathrm{L} 22 »$ au seuil $\mathrm{P}=0,05$.

Au seuil de 10 p. 100 les poids de 1000 grains des F1 monosomiques comparés à celui de la $\mathrm{F} 1$ disomique sont supérieurs pour 6 chromosomes $(3 \mathrm{~A}, 1 \mathrm{~B}, 2 \mathrm{~B}, 3 \mathrm{~B}$, $4 \mathrm{~B}, 2 \mathrm{D})$, et inférieurs pour les chromosomes $3 \mathrm{D}$ et $5 \mathrm{D}$.

Pour ce caractère la différence entre la F1 disomique et la lignée « L 22 » est significative au seuil de 5 p. 100 .

\section{DISCUSSION}

La comparaison des $21 \mathrm{~F} 1$ monosomiques de ce croisement avec la F1 disomique correspondante montre, que plusieurs chromosomes interviennent dans les relations hôte $\mathrm{x}$ parasite et pour les 2 paramètres LPI et $\mathrm{P}$ $\max$.

On peut rapprocher ces résultats de ceux qu'a obtenus JEGER (1980), également avec Septoria nodorum, en étudiant 11 paramètres de résistance partielle sur 41 génotypes de blé aussi divers que possible.

Une analyse des facteurs par la méthode de JORES KOG a permis à cet auteur de regrouper ses paramètres en quatres facteurs complexes de résistance indépendants. Il en a tiré la conclusion que si la résistance du blé à Septoria nodorum est polygénique, elle doit dépendre d'au moins quatre polygènes différents.

Dans la présente étude impliquant les génotypes de "Courtot » et de la lignée « L 22 », le tableau 2 montre que les $\mathrm{F} 1$ monosomiques $3 \mathrm{~A}, 4 \mathrm{~A}, 2 \mathrm{~B}, 5 \mathrm{~B}$ et $2 \mathrm{D}$ sont plus intéressantes que la $\mathrm{F} 1$ disomique pour les 2 paramètres considérés.

Par contre la $\mathrm{F} 1$ monosomique $7 \mathrm{D}$ est plus résistante que la $\mathrm{F} 1$ disomique pour le seul paramètre LPI, et les F1 monosomiques 5A, 6A, 1B et 7B plus résistantes pour le seul paramètre $P \max$, la valeur de l'autre paramètre n'étant pas significativement différente dans ces 2 cas.

D'autres F1 monosomiques sont au contraire plus sensibles que la $F 1$ disomique, pour les 2 paramètres (6B, 6D), pour la LPI seulement (1A, 3B, 5D), pour $\mathrm{P}$ max seulement (3D, 4D). Mais aucune inversion de la résistance n'a été observée selon le paramètre considéré.

Cela suggère l'existence d'une liaison entre LPI et $\mathrm{P}$ max, comme celle qu'avaient observée RAPILLY et al. (1984) sur feuilles en survie. C'est pourquoi les valeurs des deux paramètres obtenues pour les différentes F1 et pour la lignée « $\mathrm{L} 22$ » ont été reportées sur un graphique à double entrée (fig. 2), les valeurs étant exprimées en p. 100 des valeurs correspondantes de la F1 disomique.

Sur l'ensemble des points la droite de régression de $\mathrm{P}$ max sur LPI a pour équation :

$$
P \max =14,56+0,72 \mathrm{LPI}, r=0,57 \mathrm{HS}
$$

un tiers de la variabilité de $\mathrm{P}$ max est donc expliqué par la variabilité de LPI. 


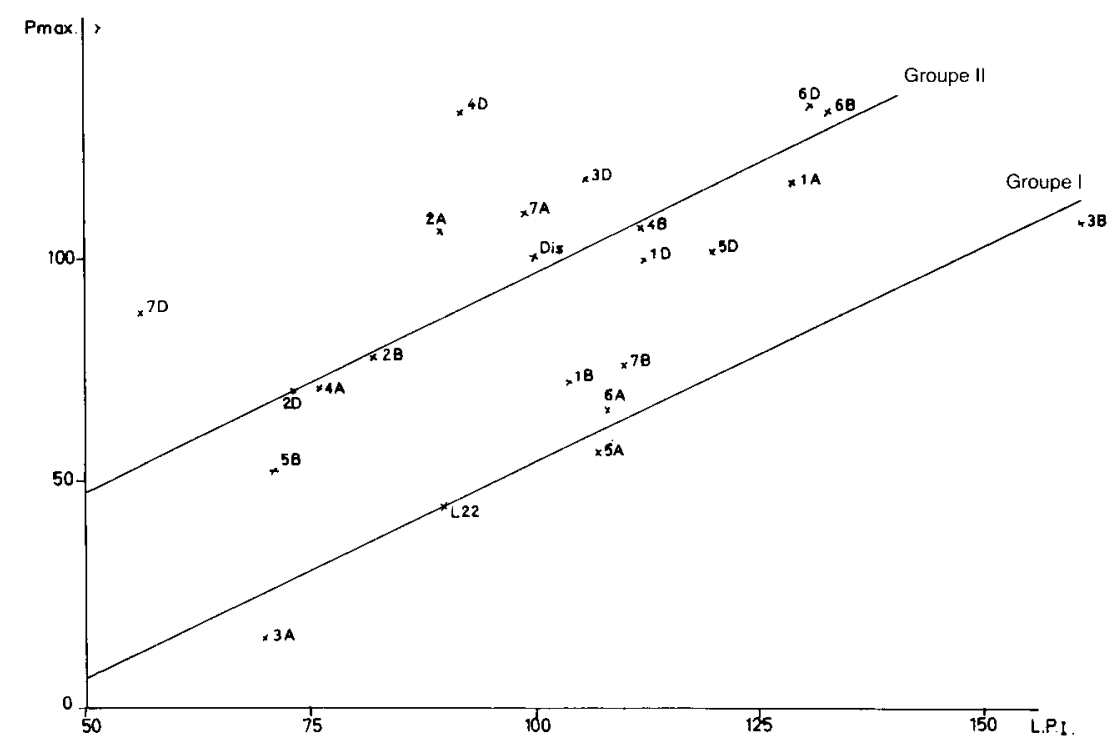

Figure 2

Relations entre LPI et P max exprimées en $p$. 100 des mêmes valeurs par rapport à la $\mathrm{Fl}$ disomique.

Pour étudier la variation de $\mathrm{P}$ max correspondant aux 2 autres tiers, LPI étant fixée, on peut distinguer sur la figure 2 trois groupes de points :

- le groupe 1 renferme les $\mathrm{F} 1$ monosomiques $3 \mathrm{~A}$, $5 \mathrm{~A}, 6 \mathrm{~A}, 1 \mathrm{~B}, 3 \mathrm{~B}, 7 \mathrm{~B}$ et la lignée « L $22 »$,

- le groupe 2 renferme les $\mathrm{F} 1$ monosomiques $1 \mathrm{~A}$, $2 \mathrm{~A}, 4 \mathrm{~A}, 7 \mathrm{~A}, 2 \mathrm{~B}, 4 \mathrm{~B}, 5 \mathrm{~B}, 6 \mathrm{~B}, 1 \mathrm{D}, 2 \mathrm{D}, 3 \mathrm{D}, 5 \mathrm{D}, 6 \mathrm{D}$ et la F1 disomique,

- le groupe 3 renferme les $\mathrm{F} 1$ disomiques $4 \mathrm{D}$ et $7 \mathrm{D}$.

A de rares exceptions près ces groupes correspondent aux diagonales du tableau 2. Les exceptions s'expliquent par le fait que dans le tableau il n'est tenu compte que de la signification des écarts, non de leur valeur absolue.

Les droites de régression correspondant aux groupes 1 et 2 sont respectivement les suivantes :

$$
\begin{aligned}
& \mathrm{P} \max =-40,23+0,96 \text { LPI }(\mathrm{r}=0,945 \mathrm{HS}), \\
& \mathrm{P} \max =\quad 0,09+0,97 \text { LPI }(\mathrm{r}=0,859 \mathrm{HS}) .
\end{aligned}
$$

Les pentes de ces 2 droites ne sont pas significativement différentes entre elles, ni avec la pente de la régression générale. Par contre la différence entre les ordonnées à l'origine de ces 2 droites est hautement significative. Elle est peu différente de l'écart pour les valeurs de $\mathrm{P}$ max entre la lignée « $\mathrm{L} 22$ » et la F1 disomique. Quant aux 2 points du groupe 3 ils définissent une droite à peu près parallèle à celles des 2 autres groupes.

Tout se passe donc comme si la part de variabilité de $P$ max liée à celle de LPI était représentée par les pentes de ces droites. Quant à la variabilité indépendante elle serait représentée par les différences entre les ordonnées à l'origine.

La liaison partielle entre les 2 paramètres pourrait être due au fait que la LPI correspond à une séquence épidémique antérieure à celle à laquelle correspond $\mathrm{P}$ max. Mais le fait qu'une telle liaison ait aussi été observée sur feuilles en survie et en conditions contrô-
Relations between LPI and P max expressed as percentages of tht same values for the disomic $F I$.

lées ne permet pas d'écarter l'hypothèse que certains gènes puissent avoir un effet pléïotropique.

Quant à la part de variabilité de $\mathrm{P}$ max indépendante de LPI, si les groupes qui ont été distingués correspondent à la réalité, ils indiquent aussi quels sont les chromosomes de la lignée « L 22 » responsables de sa résistance (groupe 1) ou de sa sensibilité (groupe 3) à ce niveau.

Et comme l'importance de $\mathrm{P}$ max est plus grande que celle de LPI dans le développement des épidémies (RAPILly \& DELHOTAL, 1986), on ne peut pas négliger le paramètre $P$ max dans la sélection de variétés résistantes.

D'autre part la présence de gènes de sensibilité chez la lignée « L 22 », indique qu'un niveau encore plus élevé de résistance doit pouvoir être obtenu.

La taille de la $\mathrm{F} 1$ monosomique $4 \mathrm{~A}$ supérieure à celle de la F1 disomique confirme que la variété «Courtot» porte le gène Rht1 (GALE \& LAW, 1976).

D'autre part, l'observation du tableau 2 ne suggère aucune liaison entre le poids de 1000 grains et les paramètres de résistance partielle à la septoriose étudiés ici.

Ces paramètres ne sont pas liés non plus à la taille ni à la précocité de floraison (tabl. 3).

\section{CONCLUSION}

Il est remarquable que cette analyse génétique confirme certains résultats obtenus par simulation, et notamment une liaison entre LPI et P max. C'est une raison de penser que cette liaison n'est pas purement fonctionnelle.

Néanmoins des différences importantes de la valeur de $\mathrm{P}$ max à LPI constante ont aussi été observées. Cette étude attire ainsi l'attention sur la nécessité de prendre le paramètre $P$ max en compte dans la sélection de variétés résistantes. 
Cependant, en raison même du nombre élevé de chromosomes concernés par ces résistances on peut s'interroger sur la part de résistance observée qui pourrait être sous la dépendance du métabolisme général des plantes, et donc en relation avec leur productivité.

On doit alors observer que la lignée «L 22 » est aussi résistante que «Carala » pour les 2 paramètres considérés, bien que sa productivité soit très supérieure. Cela permet d'espérer que la part de résistance indépendante du rendement est la plus importante dans ce matériel.
Cette étude montre enfin le parti que l'on peut espérer tirer du chromosome $3 \mathrm{~A}$ de la lignée « L 22 ». Il serait intéressant d'obtenir des lignées de substitution de ce chromosome dans différents génotypes.

Reçu le 2 mars 1987 Accepté le 17 septembre 1987.

\section{REMERCIEMENTS}

Nous remercions vivement Madame Ghania TALEB qui a effectué le dénombrement chromosomique des plantes F1.

\section{RÉFÉRENCES BIBLIOGRAPHIQUES}

Denis J. B., Bernard M., Arnoux J., Cauderon Y., 1982. Analyse des configurations méiotiques lors de la création d'une série monosomique du blé tendre «Courtot". Agronomie, 2, 701-708.

Gale M. D., Law C. N., 1976. The identification and exploitation of Norin 10 semi-dwarfing genes. PBI Ann. Report 21-35.

Jeger M. J., 1980. Multivariate models of the components of partial resistance. Protection Ecology, 2, 265-269.

Rapilly F., Delhotal P., 1986. Sur la durabilité de résistances partielles à Septoria nodorum Berk. chez le blé Triticum aestivum L., études prospectives réalisées par la simulation. Agronomie, 6, 325336.
Rapilly F., Auriau P., Laborie Y., Depatureaux C., Skajennikof M., 1981. Résistance partielle du blé, Triticum aestivum L. à Septoria nodorum Berk. Agronomie, 1, 771-782.

Rapilly F., Auriau P., Laborie Y., Depatureaux C., 1984. Recherches sur la résistance partielle du blé tendre à Septoria nodorum. Agronomie, 4, 639-651.

Sears E. R., 1954. The aneuploids of common wheat. Missouri Agric. Exp. Sta. Res. Bull 572, 59 p.

Trottet M., Merrien P., 1982. Analyse du comportement de vingt lignées de blé tendre vis-à-vis de Septoria norodum Berk. Agronomie, 2, 727-734. 